\title{
UNE RESSOURCE HALIEUTIQUE MAL CONNUE : LES GRENOUILLES. LES PROBLĖMES LIÉS A UNE VÉRITABLE GESTION DES STOCKS
}

\author{
A. NEVEU, Valérie RÉGNIER \\ Laboratoire d'Ecologie Hydrobiologique - INRA \\ 65, rue de St Brieuc - 35042 RENNES CEDEX. France
}

\section{RESUME}

Au cours de ces 20 dernières années, les Français ont consommé de plus en plus de cuisses de grenouilles importées de divers pays. Jusque vers 1960 la consommation ne concernait que la production intérieure. Il est difficile d'affirmer que la diminution des stocks sauvages a entrainé un recours aux importations. Cette diminution est à relier au développement agricole et au recul des zones humides.

Actuellement les grenouilles françaises sont protégées, leur vente est interdite et leur capture réglementée. La pêche à la grenouille est devenue une activité de loisir.

La gestion rationnelle des stocks, de même que leur développement, exige une bonne connaissance des populations, surtout au niveau du complexe Rana esculenta. La réglementation est à revoir car le plus souvent inadaptée aux problèmes locaux. Diverses propositions sont faites en ce sens et le repeuplement doit être abordé avec prudence.

\section{SUMMARY}

\section{FROGS : AN UNDERESTIMATED NATURAL RESOURCE. PROBLEMS ASSOCIATED WITH AN ACTUAL STOCKS MANAGEMENT.}

For the last 20 years the French people have eaten more and more frog legs imported from various countries. Before 1960, consumption only concerned inland production. It is difficult to say if the regression of wild stocks was the cause of imports. This regression of frog populations is in connection with agricultural development and wetlands reduction

French frogs are now protected by the law; sale of legs is prohibited and angling regulated. Frogging becomes a recreational activity.

Rational management and increasing of wild stocks require a good understanding of population dynamics, specially in Rana esculenta complex. The law is unsuitable to local context and must be reexamined. Proposals are made to this effect and restocking must be considered with much care.

\section{I - INTRODUCTION}

La consommation de cuisses de grenouilles fait partie de l'art culinaire traditionnel. Plat intégré à l'origine au folklore de certaines régions riches en marais et étangs, il s'est ensuite fait connaître dans les grandes villes avec le développement des transports et des moyens de conservation. Dans tous les cas, il est resté plutôt une denrée de luxe dans bien des esprits, à l'affiche des meilleurs restaurants ou ayant place à l'occasion de fêtes, surtout en fin d'année. Ceci d'autant plus que la consommation s'est développée avec celle des surgelés, phénomène que l'on retrouve dans d'autres pays européens (RFA, Belgique...) et aux USA. II faut noter que contrairement aux idées recues, les Français n'ont pas le monopole de cette consommation, la majorité des pays latino-américains et certains pays d'Extrême-Orient en consomment aussi.

\section{2 - RÉgIONS ET ESPECES EXPLOITEES}

En France, les principales zones d'exploitation traditionnelle étaient limitées à la façade ailantique (de Redon à Bordeaux), aux Dombes et au Ried alsacien.

La consommation traditionnelle porte sur 2 types de grenouilles : d'une part les grenouilles vertes, d'autre part les grenouilles rousses.

Les grenouilles vertes appartiennent au complexe Rana esculenta L. (" grenouille comestible ") ; ce sont des animaux essentiellement aquatiques au cours de leur vie larvaire et adulte. Elles peuplent tous les types de plans d'eau avec une préférence pour les étangs riches en certains végétaux, les mares, les marais. La ponte a lieu à la fin du printemps et donne lieu à des rassemble- 
ments importants de mâles qui chantent en phase formant un " chorus " pour appeler et stimuler les femelles; c'est une période où la pêche est facilitée, la méfiance est réduite et l'agressivité maximum. Il y a ensuite un passage progressif de cette répartition très agrégative à une répartition plus uniforme pour la croissance estivale. Fin septembre début octobre, I'hibernation commence (dans la vase, les berges, les végétaux) et se poursuit jusqu'en avril.

Les grenouilles rousses appartiennent à 3 espèces: Rana temporaria L., $R$. dalmatina Bonaparte et $R$. arvalis Nilsson. Seule la première est exploitée traditionnellement, c'est " la grenouille des bois " par opposition aux deux autres appelées " grenouilles des champs ". Rana temporaria est une espèce terrestre a l'état adulte; elle rejoint l'eau pour hiberner et frayer, ce qui donne lieu aussi à des rassemblements importants avec, cependant, une activité plus discrète et nocturne. La fraie a lieu au premier radoucissement humide $\left(8\right.$ a $\left.10^{\circ}\right)$; pour la Bretagne, les pontes s'observent dès le 15 janvier jusqu'au 15 février la plupart du temps, sauf en cas de gel, celui-ci bloquant momentanément l'activité. En montagne, la ponte suit le début de la fonte des neiges; la période de fraie est donc fonction de l'altitude et de la latitude (jusqu'au cercle polaire). Cette période est aussi la plus sensible, d'une part parce qu'elle donne lieu à des migrations nocturnes notoires des zones d'hibernation vers les frayères - c'est le moment où beaucoup de géniteurs se font écraser sur les routes - d'autre part, parce que les rassemblements sont denses et la récolte facile. Après le dépôt des œufs, la dispersion des animaux est rapide, soit en milieu forestier (surtout en plaine), soit en prairie (en montagne); ils deviennent alors beaucoup moins vulnérables.

Si la ponte des grenouilles vertes s'effectue dans des masses d'eau relativement importantes et permanentes (sauf certaines zones d'inondation et marais), les grenouilles des bois pondent souvent dans de simples flaques, des ornières qui peuvent s'assécher rapidement. En forêt de Rennes, plus de $50 \%$ des frayères sont à sec avant le début de la métamorphose, la survie des têtards restants ne dépasse pas quelques pour mille.

Pour la grenouille rousse les zones de récolte sont donc plus dispersées et recouvrent principalement les pays de montagne (Massif Central, Jura, Ardennes...). Du reste, sa chair plus jaune est surtout appréciée au niveau local.

\section{3 - PRINCIPALES METHODES DE CAPTURE}

Les méthodes traditionnelles de capture restent très archaïques ; elles varient suivant l'espèce et la saison, la plupart sont illégales.

Pour les grenouilles vertes on parle plus volontiers de "pêche ", puisque la méthode la plus courante est effectivement la pêche à la ligne, soit avec le traditionnel bout de tissu rouge, soit de plus en plus avec une boulette de bas de nylon qui accroche les petites dents des grenouilles; dans ce cas I'hameçon n'est pas nécessaire et les animaux n'étant pas blessés peuvent être stockés. Cette pêche " à roder " est surtout efficace au moment des grands rassemblements pour la fraie. La pêche avec un hameçon triple est aussi très pratiquée; elle permet d'harponner un grand nombre d'animaux au moment des chorus et elle prend plus de mâles que de femelles. Ce mode de capture a l'inconvénient de blesser les animaux et les cuisses doivent être prélevées rapidement. D'autres méthodes sont plus rarement employées comme la pêche aux esches naturelles (sauterelles, vers..) montées sur hameçon simple, ou encore la pose de nombreux hameçons triples sur les frayères où les grenouilles s'empalent d'elles-mêmes, sans parler de l'emploi de divers filets (haveneaux, carrelets, etc.).

Pour les grenouilles rousses il s'agit plus d'un acte de "chasse". La récolte s'effectue au moment de la reproduction, à la main, éventuellement avec une épuisette, très généralement la nuit à l'aide d'une lumière qui tend à immobiliser l'animal. Localement, il est possible de déterminer les lieux d'hibernation et de récolter des animaux hibernants profitant de leur inactivité et d'une certaine concentration.

Dans tous les cas, il faut reconnaître que l'efficacité des différentes méthodes reste assez limitée si l'on considère un objectif de pêche commerciale; la pêche à la grenouille est devenue progressivement une activité de loisir faute de rentabilité.

\section{4 - INTÉRET ÉCONOMIQUE}

La réglementation actuelle n'autorise que la pêche " familiale " d'autoconsommation ; il est donc très difficile d'évaluer les quantités récoltées. On peut avancer le chiff́re de 50 à $100 \mathrm{~T} / \mathrm{an}$ d'après les captures de 1977 (FLEURY et L'HOSTIS, 1977), mais cette estimation faite à partir des données des grossistes de l'époque reste très contestable, de même que la récolte de 600 à $700 \mathrm{~T} / \mathrm{an} 20$ ans auparavant.

L'examen des statistiques douanières de ces 20 dernières années montre une croissance rapide des importations (le déficit de la balance commerciale atteint 125 millions de F en 1983, c'est le $2^{\mathrm{e}}$ poste déficitaire en produits d'eau douce, après le saumon). De 1962 à 1970, les importations de 
grenouilles vivantes et de cuisses surgelées (ou congelées) progressent au même rythme, celles d'animaux vivants se stabilisent (autour de $700 \mathrm{~T} / \mathrm{an}$ ) alors que celles de surgelés augmentent fortement (Fig. 1). Si ce dernier produit peut être reconnu comme nouveau, avec un développement indépendant des besoins traditionnels, on peut considérer que le développement de la filière grenouilles vivantes correspond au manque de grenouilles autochtones.

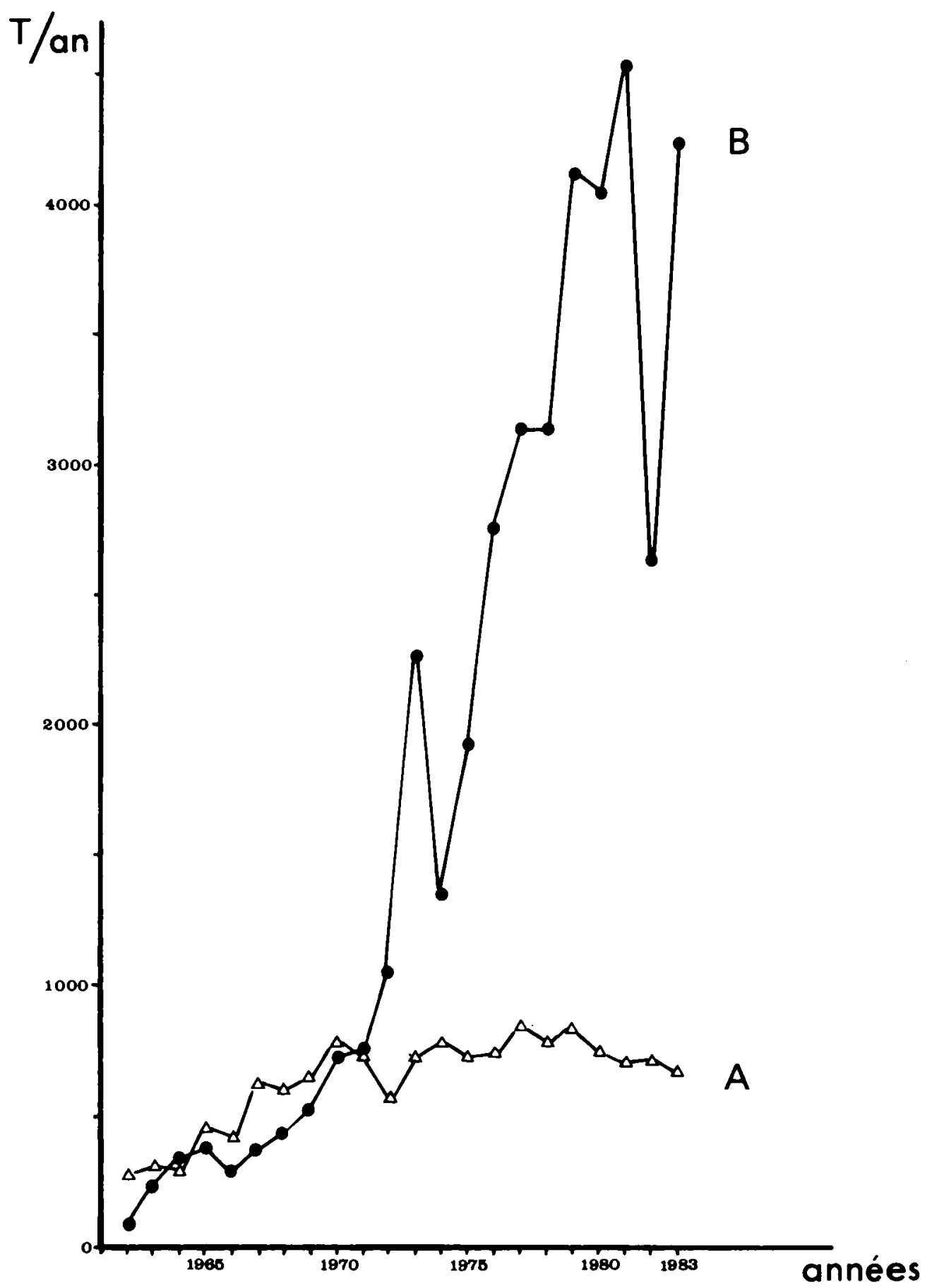

Figure 1: Evolution des importations de grenouilles ces 20 derniéres années (statistiques douanières)

A / Importations de grenouilles vivantes

B / Importations de cuisses de grenouilles congelées et surgelées.

Figure 1 : Evolution of frogs imports during the last 20 years (Customs data)

A/ Imports of live frogs

B/ Imports of freezed and deep-freezed frog legs. 
En effet, la chaine grenouilles vivantes est assez particulière ; elle ne concerne que quelques grossistes spécialisés dans la préparation des cuisses, surtout dans la région lyonnaise; elle ne concerne pas (ou peu) directement le consommateur mais avant tout les restaurateurs. Il s'agit aussi d'animaux de petite taille, d'espèces européennes ou méditerranéennes (Turquie. Egypte, Albanie. Bulgarie, etc.) alors que la plupart des surgelés sont constitués de grosses espèces subtropicales (Indonésie. Indes, etc.). II semblerait cependant que les petites espèces soient plus appréciées des Français (FLEURY et L'HOSTIS, 1977)

Du reste, sile prix de vente des cuisses surgelées reste modéré $(40-60 \mathrm{~F} / \mathrm{kg})$, celui des fraîches est en fait un met luxueux $(90-120 \mathrm{~F} / \mathrm{kg}$ ) pour le consommateur. Tout ceci peut expliquer la stabilisation des importations de grenouilles vivantes depuis 1970 alors que les cuisses surgelées ont une croissance régulière depuis 1962, de type exponentiel, parallèlement au phénomène général du développement de la consommation de divers produits, particulièrement des surgelés. Enfin, il faut remarquer que pour comparer l'importance relative des deux filières, le tonnage en grenouilles vivantes doit être divisé par 2 environ, dans la mesure où les cuisses représentent approximativement la moitié du poids total. Il est traditionnel de classer les cuisses suivant trois grandes catégories: les grosses ( $2 \mathrm{dz} / \mathrm{kg}$ ), les movennes ( $4 \mathrm{dz} / \mathrm{kg}$ ), les petites $(7-8 \mathrm{dz} / \mathrm{kg}$ ).

En conclusion, sur le plan économique, la pêche à la grenouille est donc devenue une activité de loisir dont l'intérêt est à relier à celui de la pêche à la ligne en général et au développement du tourisme. La compensation, ne serait-ce que partielle, des importations ne peut se concevoir sans la mise en place d'élevages (sous diverses formes à définir) dont la technologie reste à découvrir.

\section{5 - ETAT ACTUEL DES STOCKS}

En première analyse il semble donc que le recours aux importations se soit produit à la suite du déclin général de ces animaux. Cette régression est difficile à estimer; on peut seulement remarquer l'analogie entre les chiffres estimés de production autochtone il y a 20 ans (600-700 T/an) et ceux des importations de grenouilles vivantes depuis 1970

Dans l'ouest il est courant de rencontrer d'anciens pêcheurs de grenouilles vertes qui capturaient $2.000 \mathrm{ind} . / \mathrm{J}$, alors qu'actuellement 200-300 individus représentent un score honorable. De même, au Luxembourg GOSSEYE (1979) signale une chute des captures de grenouilles rousses depuis 20 ans. 11 faut cependant noter qu'en 1982 un grand quotidien de l'ouest vantait le savoir-faire d'un pêcheur vendéen qui capturait encore 1.500-2.000 ind./J.

Il est certain que d'une manière générale, les populations ont régressé dans la mesure où leur habitat préférentiel est lui-même en recul (marais). Si les données concernant la France restent très rares (BAUDOUIN-BODIN et MAILLARD, 1972 ; MARION, 1975) plusieurs études montrent une régression des batraciens (surtout grenouilles des bois et crapauds) au niveau européen qui aurait débuté, pour l'Angleterre, entre les années 1950 et 1960 (BEEBEE, 1973, 1977 ; BEEBEE et GRIFFIN, 1977 : COOKE et FERGUSON, 1976 ; ILOSWAY, 1977 ; OBERT, 1977 ; SIMMS, 1969). II faut noter que ces dates correspondent aux toutes premières importations de grenouilles en France qui se situeraient vers 1954 d'après FLEURY et L'HOSTIS (1977). Les grenouilles vertes, espèces les plus répandues en Europe, seraient aussi concernées (LEUTSCHER, 1975 ; GROSSENBACHER, 1977).

Les causes de cette régression sont multiples

- réduction des zones humides par le drainage, la protection contre les crues (assèchement du Ried alsacien), le développement des cultures au détriment des pâtures :

- pour les rousses, réduction des massifs forestiers, urbanisation et développement des routes, véritables pièges à géniteurs en migration (GOSSEYE, 1977 ; TERHIVUO, 1981):

- développement d'une agriculture industrielle mécanisée et polluante (destruction des grenouilles rousses par les engins, arasement des haies, eutrophisation des eaux,...);

- impact des pesticides surtout au niveau larvaire (insecticides dans les Dombes, lutte antimoustiques dans le Languedoc). Des travaux récents montrent que les batraciens sont rares dans les mares de zones de cultures intensives du Bassin Parisien en liaison avec une baisse générale de la diversité de la faune (MARCHAL-SEGAULT et RAMADE, 1981 ; ECHAUBARD, com. pers.). De nombreuses études consacrées aux pesticides agricoles montrent leur toxicité pour les batraciens (COOKE, 1970, 1973, 1974a, 1977, 1979, 1981 ; HALL et KOLBE, 1980 ; LAUGHLIN et LINDEN, 1982 ; LYONS et al., 1976 ; MARCHAL-SEGAULT, 1976 ; MARCHAL-SEGAULT et RAMADE, 1981 ; PAULOV, 1977, 1981 ; WEIS, 1975)

Cette toxicité peut agir

- directement sur les populations d'amphibiens par une augmentation de la mortalité chez les jeunes stades, un retard de développement, des malformations (tératogénèse), des modifications du comportement ;

- indirectement : des herbicides comme le diquat ou le dichlobenil n'ont pas d'effet toxique direct 
aux doses employées en agriculture, mais ils détruisent une végétation indispensable en tant que support de nourriture, couvert et/ou sites de fraie (COOKE, 1977).

- La prédation humaine a peut-être conduit à une certaine surexploitation de stocks par ailleurs affaiblis. Ce phénomène a été montré aux USA sur Rana pipiens, espèce très utilisée aussi bien pour la recherche et l'enseignement que pour la consommation (GIBBS et al., 1971). Pour la France cet impact est difficile à cerner : I'on accuse souvent le développement du tourisme sur la façade atlantique. L'absence d'une réglementation adaptée a peut-être favorisé un ramassage abusif de grenouilles rousses en certaines localités. La Yougoslavie, qui était un de nos plus fidèles fournisseurs en grenouilles vivantes jusqu'en 1977 (en moyenne $200 \mathrm{~T}$ /an), ne fournit plus que 20 à $30 \mathrm{~T}$ /an en liaison avec la baisse des stocks probablement trop exploités.

- Enfin, pour les grenouilles vertes, un problème particulier de génétique, conduisant à une stérilité de certains croisements, a pu favoriser notablement cette régression. C'est un point important, complexe, qu'il est utile d'examiner plus en détail.

\section{6 - STRUCTURE SPÉCIFIQUE DES POPULATIONS DE GRENOUILLES VERTES, SON INCIDENCE ECOLOGIQUE}

C'est en Pologne que BERGER $(1964,1966)$ fut le premier à montrer la présence de trois types morphologiques de la grenouille verte : $R$. lessonae CAM., $R$. esculenta L. et $R$. ridibunda PALL., qui ne correspondaient pas à de simples variations géographiques, mais constituaient des " espèces" différentes.

Des expériences de croisements artificiels (Tab. 1) lui ont permis d'aboutir aux résultats suivants : $R$. ridibunda et $R$. lessonae sont bien de " bonnes " espèces à l'identité génétique bien définie et $R$. esculenta est leur hybride (BERGER, 1967, 1968a, 1970, 1971a, b, c, 1973a et b).

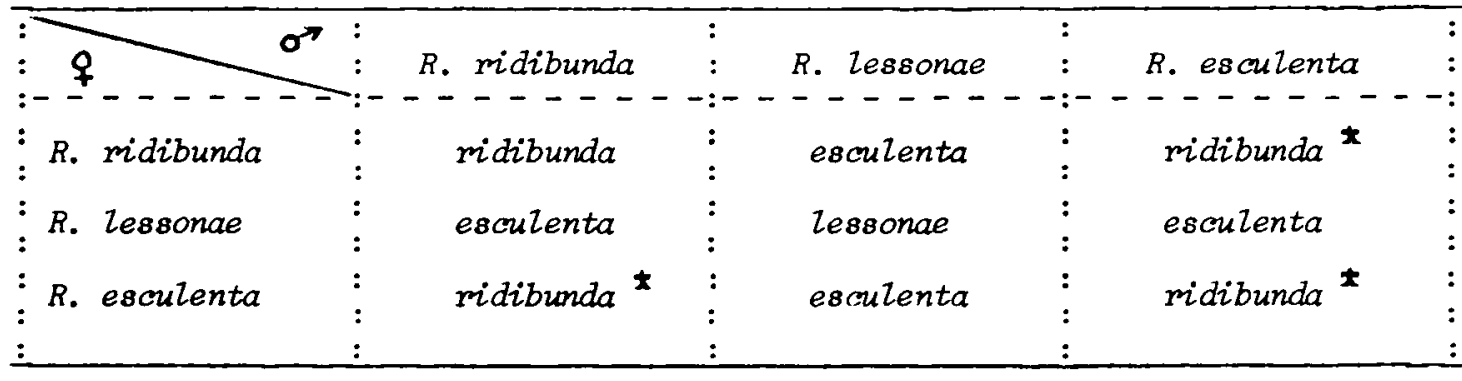

* forte mortalité des larves

Tableau 1 : Phénotypes obtenus par BERGER à la suite de différents croisements entre $R$. ridibunda, $R$. lessonae et $R$. esculenta.

Tableau 1 : BERGER'S phenotypes obtained after different crossing between $\boldsymbol{R}$. ridibunda, $\boldsymbol{R}$. lessonae and $R$. esculenta.

Les différences morphologiques minimes trouvées entre les trois formes s'accompagnent de différences écologiques (Tab. 2). II faut remarquer que la maturité retardée de $R$. esculenta est compensée par une plus grande fertilité à poids égal (REGNIER, 1983).

Les hypothèses de BERGER, à l'origine, très controversées, furent le point de départ de nombreuses recherches en Europe (BLANKENHORN, 1973 ; BLANKENHORN et al., 1971. 1973 ; GUNTHER, 1973). Elles ont été confir mées surtout par l'utilisation de méthodes biochimiques (analyse de protéines sériques et d'enzymes par électrophorèsel: les trois formes de grenouilles vertes présentent en effet des phénotypes électrophorétiques différents pour la plupart des protéines étudiées et $R$. esculenta possède dans tous les cas un phénotype d'hybride (ENGELMAN, 1972, 1973. 1974 ; TUNNER, 1970, 1972, 1973a et b : UZZELL et BERGER, 1975 ; VOGEL et CHEN, 1976).

Par la suite, les divers travaux menés dans ce domaine ont abouti à la découverte de phénomènes de plus en plus complexes et parfois surprenants

- fertilité moindre chez les mâles $R$. esculenta, se traduisant par des anomalies à la méiose lors de la formation des gamètes (GUNTHER, 1975a, 1979), d'où la stérilité presque totale des autocroisements :

- paradoxalement, des populations pures $R$. esculenta ont été observées et sont donc capables de subsister et de se reproduire (GUNTHER, 1975b : KNUDSEN et SCHEEL, 1975). 


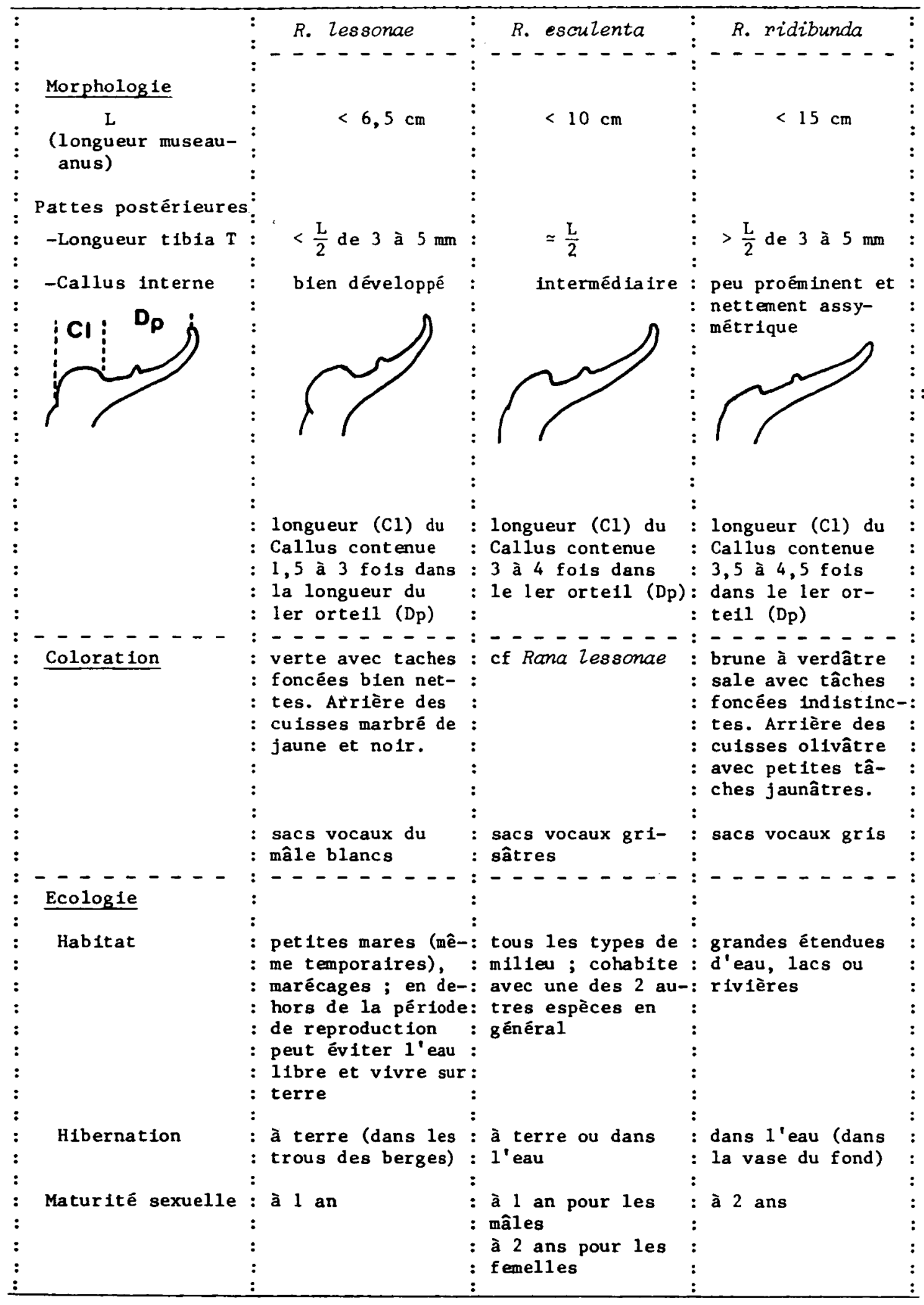

Tableau 2: Principaux caractères distinctifs des trois formes de grenouilles vertes $(R$. ridibunda, $R$. lessonae, $R$. esculenta).

Tableau 2 : Main characters to separate the three morphological forms of green frogs $(R$. ridibunda, $R$. lessonae, $R$. esculenta). 
En fait, les grenouilles de phénotype esculenta sont des hybrides d'un type un peu particulier puisqu'elles peuvent se maintenir dans des populations où une seule espèce parentale $(R$. lessonae ou $R$. ridibunda) est présente ; de plus, elles conservent le statut d'hybride $F$, de génér ation en génération (il n'y aurait donc pas de recombinaisons entre gènes parentaux).

Ce mécanisme, ou hybridogénèse, surprenant au niveau génétique, pourrait s'expliquer par l'élimination du génome d'un des parents lors de la formation des gamètes chez $R$. esculenta, ce qui assure une transmission clonale des caractères (TUNNER, 1974).

Cette gamétogénèse de type hybridogénétique qui se produit chez $R$. esculenta (pour les deux sexes) a été décrite de nombreuses fois à travers toute l'Europe.

L'utilisation de méthodes biochimiques de plus en plus sophistiquées ont permis de progresser encore dans la systématique des grenouilles vertes avec la mise en évidence

- de lignées triploïdes $R$. esculenta dans certaines populations (BERGER, 1979 ; BERGER et ROGUSKI, 1978 ; GUNTHER, 1970, $1975 \mathrm{~b}$; GUNTHER et al., 1979 ; OGIELSKA-NOWAK, 1978 ; UZZELL et BERGER, 1975 ; UZZELL et al., 1975). De nombreux triploïdes ont été trouvés en Bretagne (REGNIER, 1983) :

- de quelques cas de recombinaisons entre génomes lessonae et ridibunda au sein de populations mixtes $R$. ridibunda - $R$. esculenta (GUNTHER, 1975a ; GUNTHER et HAHNEL, 1976 ; KOREFSANTIBANEZ, 1979 ; UZZELL et al., 1977) ;

- de formes morphologiques différentes des trois habituellement observées, formes à élever au rang d'espèces :

- $R$. perezi, espèce voisine de $R$. ridibunda, présente en Espagne et dans le Sud de la France (GRAF et al., 1977 ; HOTZ, 1974 ; TUNNER et UZZELL, 1974 ; WIJNANDS, 1978) ;

- espèce du sud de l'Italie, proche de $R$. lessonae (UZZELL, 1979 ; UZZELL et HOTZ, 1979):

- deux espèces dans la péninsule balkanique (sud-ouest de la Yougoslavie et nord-ouest de la Grèce) assez proches de $R$. lessonae (HOTZ et UZZELL, 1982, 1983 ; TUNNER et HEPPICH, 1982).

Actuellement, parmi les lignées européennes de $R$. esculenta, on distingue - deux types de lignées diploïdes

- deux types de lignées triploïdes (UZZELL et HOTZ, 1979 ; UZZELL et al., 1980).

- Les diploïdes (possédant un génome esculenta) cohabitent avec une espèce parentale dont elles dépendent lors de la reproduction (du fait du processus d’hybridogénèse) et qui leur fournit le génome perdu durant la gamétogénèse. On a ainsi

- des populations $R$. lessonae - $R$. esculenta lou système L-E : UZZELL et BERGER, 1975 ; UZZELLet al. 1977). Dans ce cas, c'est le génome lessonae qui est éliminé à la gamétogénèse. Ces populations sont les plus communes en Europe et particulièrement en France.

- des populations $R$. ridibunda - $R$. esculenta (système R-E). Les deux mécanismes (élimination du génome lessonae ou génome ridibunda) sont alors possibles (UZZELL et al., 1977).

- Les triploïdes peuvent être de deux types :

- LLR (2 génomes lessonae, 1 génome ridibunda):

- ou RRL ( 2 génomes ridibunda, 1 génome lessonae) (GUNTHER 1975b ; GUNTHER et al., 1979 ; UZZELL et al. . 1975) et se rencontrent fréquemment parmi les populations pures $R$. esculenta.

Les lignées hybridogénétiques $R$. esculenta (provenant à l'origine de l'hybridation $R$. lessonaex $R$. ridibunda) ne sont pas les seules lignées de ce type en Europe puisque l'on a découvert récemment deux nouveaux hybrides hybridogénétiques, I'un en Italie (UZZELL et HOTZ, 1979), I'autre en Espagne et dans le sud de la France (GRAF et al., 1977), (Tab. 3).

Les recherches actuelles tentent de découvrir les facteurs qui contrôlent le processus d'hybridogénèse (BERGER, 1983 ; BINKERT et al., 1982 ; GRAF et MULLER, 1979 ; HEPPICH et al., 1982 ; HOTZ et UZZELL, 1983 ; TUNNER et HEPPICH, 1981 ; UZZELL et al., 1980).

Le mécanisme évolutif qui a conduit à la spéciation des grenouilles vertes européennes peut se résumer ainsi (BERGER, 1973a ; HOTZ, 1974 ; UZZELL et BERGER, 1975):

Les diverses espèces se seraient différenciées durant les glaciations du pléistocène (recul général de la faune dans diverses régions refuge) puis, après la dernière glaciation (Würm), ces régions refuge ont servi de point de départ à l'extension des populations qui a abouti à une recolonisation des régions plus septentrionales.

Les différentes espèces seraient alors devenues largement sympatriques en Europe, des hybridations auraient donc donné naissance aux lignées hybrides ( $R$. esculenta, autres hybrides).

Cependant, il est possible que la divergence entre $R$. lessonae et $R$. ridibunda soit intervenue bien avant les glaciations du pléistocène; des hybridations auraient alors pu se produire ainsi que pendant chaque période interglaciaire. On aurait donc plusieurs lignées $R$. esculenta, les unes antérieures au pléistocène, les autres postérieures à la glaciation Würm (UZZELL, 1978). 


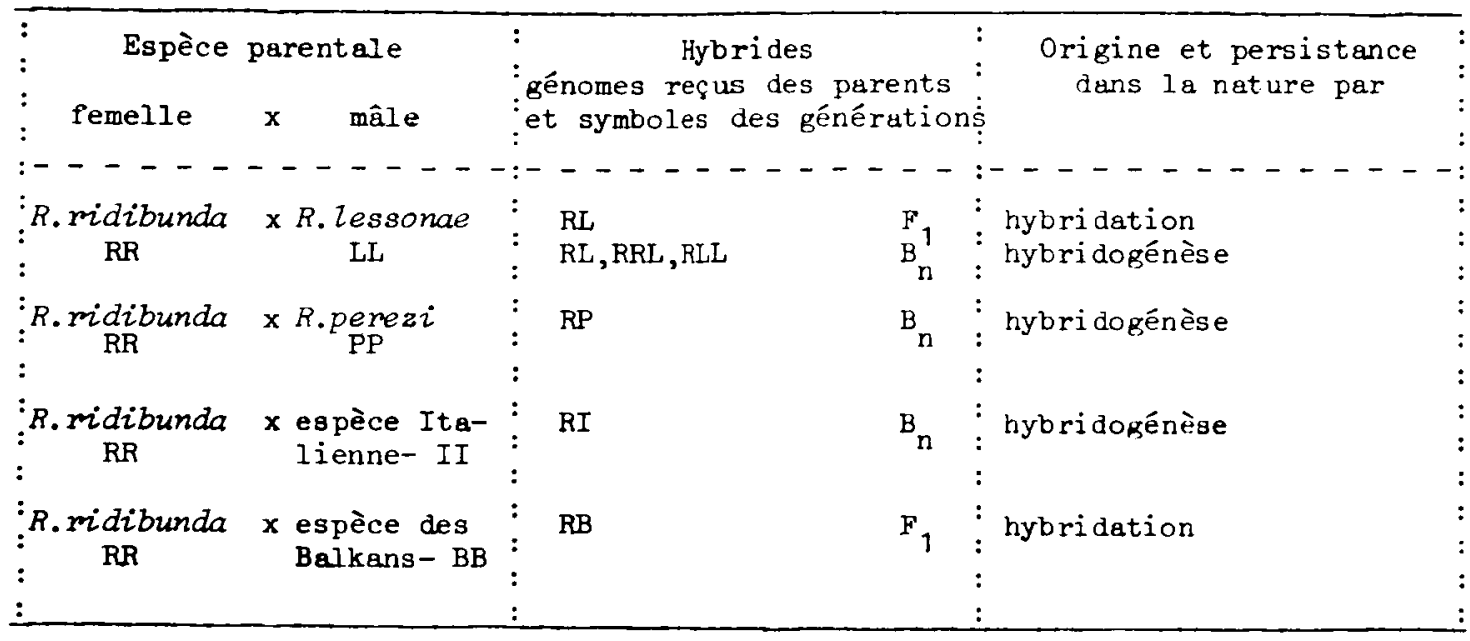

$F_{1}$ : première génération d'hybrides

$B_{n}$ : différentes générations d'hybrides obtenus par croisements en retour

Tableau 3 : Les cinq espèces de grenouilles vertes européennes et leurs hybrides (d'après BERGER, 1983).

Tableau 3 : The five species of green frogs in Europe and their hybrids (after BERGER, 1983).

A l'heure actuelle, dans la nature, il ne se formerait plus guère d'hybrides $F_{1} R$. esculenta, compte tenu de la divergence (Tab. 2) entre les modes de vie et les morphologies des espèces $R$. lessonae et $R$. ridibunda (conséquence probable d'une forte pression sélective visant à réduire ou supprimer les croisements entre ces deux formes, étant donné la stérilité presque totale des hybrides).

Pour résumer la situation française et compte tenu des connaissances actuelles assez limitées. il semblerait qu' au nord d'une ligne Bordeaux-Nice l'on ait des populations $R$. lessonae associées à son hybride $R$. esculenta qui peut éventuellement être seul et présenter des triploïdes. Au sud $R$. perezi dominerait avec éventuellement son hybride (" grenouille de Graf ") connu pour l'instant du Gard. Par ailleurs, il existe quelques îlots de $R$. ridibunda sur la côte ouest à la suite de fuites d'importateurs. En Corse seule la grenouille" italienne du Sud " serait présente. Cette situation demande des compléments d'information à partir d'analyses sérologiques précises.

Pour conclure, en ce qui concerne le déclin des populations, une étude récente (REGNIER, 1983) montre que $R$. esculenta présente bien une certaine vigueur hybride, en particulier par une meilleure résistance à l'eutrophisation, une capacité migratoire plus grande qui permet la colonisation de milieux neufs et le maintien dans des biotopes uniformisés. Par contre, les milieux colonisés par $R$. lessonae (mares, marais...) sont plus "sauvages " et sont en régression face au développement agricole (drainage, lutte anti-crues). Les populations de $R$. lessonae sont donc plus sensibles à cette évolution et leur recul pourrait être la cause d'une baisse de fertilité par le développement d'autocroisements de $R$. esculenta très généralement stériles.

\section{7 - REgLEMENTATION}

Diverses mesures de protection ont été prises pour enrayer le déclin des populations :

- Loi no 75.629 du 10 juillet 1976 relative à la protection de la nature (art. 3 et 4) suivie du décret d'application no 77.1295 du 25 novembre 1977 ;

- Arrêté du 24 avril 1979 fixant la liste des Amphibiens et Reptiles protégés sur l'ensemble du territoire, modifié par l'arrèté du 6 mai 1980 .

Cette réglementation peut se résumer aux faits suivants

- toutes les espèces de grenouilles (Rana sp.) sont protégées à l'exclusion de la grenouille verte (Rana esculenta) et de la grenouille rousse (Rana temporaria) à l'état adulte. En effet, les cufs et les têtards sont totalement protégés : leur ramassage est donc interdit ;

- quelle que soit l'espèce, sont interdits : Ia mutilation, la naturalisation, le colportage, la vente ou l'achat de spécimens vivants ou morts.

Par conséquent, seule une pêche" familiale " de la grenouille verte et de la rousse est tolérée 
A ceci s'ajoutent, pour les eaux libres, l'assimilation de la grenouille au poisson et l'application de la réglementation générale de la pêche fluviale.

Dans les conditions actuelles cette réglementation est mal appliquée :

- assimiler les grenouilles au poisson conduit à l'interdiction de la pêche d'harponnage avec des triples, de la pêche à la main, de la pêche de nuit avec des lumières ou encore de l'emploi d'épuisettes, sans parler du ratissage des rousses sur leurs frayères avec des rateaux:

- le respect des dates de fermeture est plus que superficiel car souvent ces dates sont méconnues :

- la vente des captures aux restaurateurs reste une pratique courante volontairement ou non :

- la pêche s'effectue très souvent sans aucun permis.

Très généralement la pêche à la grenouille est considérée comme une activité traditionnelle sans contrainte, comme.le ramassage des champignons ou des escargots. La plupart des individus qui la pratiquent sont totalement ignorants de la réglementation.

Mais il faut aussi reconnaître que la réglementation actuelle est mal adaptée, par exemple au niveau des dates de fermeture de la pêche.

Les dates de fermeture générale du poisson ne protègent que partiellement la reproduction des grenouilles, celles-ci n'ayant rien à voir avec le classement catégoriel des cours d'eau.

Ainsi, en 1983, la période de fermeture $2^{\mathrm{e}}$ catégorie s'étendait du 20 avril au 11 juin ; on pouvait donc pêcher les rousses de janvier à avril en pleine reproduction et les vertes au milieu de leur période de ponte (pour l'ouest). Inversement, si la fermeture en $1^{\text {e }}$ catégorie, qui se termine en mars, protège la reproduction des rousses, elle ne protège pas celle des vertes. Il faut cependant reconnaître que la zone à salmonides coïncide en partie avec celle à grenouilles rousses, surtout en altitude. II y a aussi des différences dans l'interprétation de ces fermetures. En 1983, si l'on en croit les données de la “ Gazette Officielle de la Pêche " (n० 736 et 738), les départements de Loire Atlantique, Paris, Vendée, Hauts de Seine et Seine Saint-Denis n'auraient pas de $1^{\text {ere }}$ catégorie et la pêche aux grenouilles pourrait donc se dérouler du 12.06 .83 au 19.04.84. Plus surprenant, la Corse et le Loir et Cher n'auraient pas de fermeture pour les grenouilles. Quant au Lot-et-Garonne, à la Marne et au Var, ils autorisent l'exercice de la pêche à la ligne en $2^{\circ}$ catégorie pendant la période de fermeture générale (20.04. au 11.06), donc logiquement des grenouilles (en pleine reproduction des vertes !).

II reste le problème de l'adéquation des périodes de fermeture au climat local ; il est anormal que les dates soient les mêmes dans les Ardennes que dans l'Aude, ou en Vendée qu'en Haute-Savoie. Si les grenouilles vertes, animaux surtout de plaine, ont une reproduction en mai-juin pour pratiquement toute la France (exception 1984 : début ponte en avril), par contre les rousses présentent de grandes variations depuis mi-janvier en Bretagne jusqu'à juillet pour la haute-montagne.

Si l'on applique à la lettre l'arrêté de 1980 il ne faudrait capturer que l'hybride $R$. esculenta parmi les grenouilles vertes. Mais comment reconnaître l'espèce au niveau du pêcheur puisque seule l'analyse sérologique est efficace ? De même, pour les grenouilles rousses, il n'est pas évident de séparer $R$. temporaria de dalmatina pour une personne non entraînée.

Se pose aussi le problème des " eaux closes "où le propriétaire est libre de pêcher à sa guise les poissons présents qui lui appartiennent pleinement, tant qu'il respecte le Code Pénal sur les droits de l'animal. Mais il faut noter que si les poissons ne peuvent s'échapper par la voie terrestre ce n'est pas le cas des grenouilles qui migrent volontiers; elles se rapprochent alors du cas du gibier à poils. II serait donc souhaitable d'appliquer une partie du Code Rural concernant le gibier, par exemple autoriser une pêche libre uniquement si la propriété est entourée efficacement, interdisant les échanges extérieurs (murs, grillage à mailles au maximum de $0.5 \mathrm{~cm}$ avec rabat).

En conclusion, il serait temps de mettre au point une réglementation spécifique à la grenouille (comme c'est le cas pour les écrevisses), indépendante de celle du poisson. Par exemple

- fermeture de la pêche aux grenouilles vertes du $1{ }^{\text {er }}$ avril au 30.juin pour éviter toute pêche abusive sur les rassemblements de reproducteurs :

- fermeture de la pêche aux grenouilles rousses adaptée au climat local (de janvier-février pour la côte atlantique, à juin-juillet pour la haute montagne) :

- n'autoriser que la pêche à la ligne, de jour, sans triple.

Ces quelques mesures, si elles étaient respectées, protègeraient presque totalement les grenouilles rousses qui ne se capturent pas à la ligne, et assureraient la pérennité des vertes.

\section{8 - CONCLUSIONS GENERALES - PROSPECTIVES}

Les grenouilles, si connues tant sur le plan culinaire que physiologique, sont en fait à découvrir au niveau de l'écologie, de la dynamique des populations et de l'évolution des peuplements. Qu'il y ait déclin pour de multiples raisons, c'est probable, encore faudrait-il nuancer suivant les régions, aucune donnée sérieuse n'étant disponible. 
Doit-on protéger totalement ces batraciens en interdisant toute pêche ? Ce n'est pas évident car si la protection d'homéothermes à faible taux de reproduction, à maturité sexuelle tardive (rapaces, grands mammifères) peut être efficace, autant celle de poïkilothermes à fort taux de reproduction est souvent illusoire (poissons, batraciens...) en rapport avec une plus grande dépendance du milieu. La meilleure protection des grenouilles passe par la sauvegarde de certains biotopes favorables (marais), surtout des frayères. Pour les grenouilles vertes se pose le problème du maintien de la fertilité par $R$. lessonae. On peut envisager la protection de ses sites et la fixation d'une taille légale de capture entre 60 et $70 \mathrm{~mm}$ (longueur museau-anus) qui la protégerait indirectement en rapport avec sa taille plus faible.

En fait, de nombreuses études sont à faire pour arriver à un esprit de gestion des stocks, c'est-à-dire gérer un véritable capital dont on n'exploite que les intérêts. Pour cela, il faut avant tout connaître la structure des populations, leurs taux de renouvellement, l'influence du milieu pour définir des quotas d'exploitation (intérêts) sans dommage pour le stock global (capital). Des premiers résultats dans l'ouest concernant les grenouilles vertes montrent une relative stabilité des populations (malgré la pêche) avec un taux de renouvellement rapide, la plupart des individus ne pondant qu'une seule fois (REGNIER, 1983). De tels peuplements sont le signe d'une haute productivité qui pourraient permettre une exploitation rationnelle modulée. Par contre, les grenouilles rousses d'altitude, à développement lent, à maturité tardive, sont plus sensibles à un risque de surexploitation locale

La réaction devant la situation actuelle peut être aussi de repeupler les zones " autrefois" fertiles. A ce niveau de nombreux problèmes d'élevage sont à résoudre (NEVEU, 1980). S'il existe aux USA quelques élevages de grenouilles sophistiqués (utilisant beaucoup d'antibiotiques), ils ne sont pas rentables pour la consommation et ne commercialisent que des animaux expérimentaux pour les laboratoires à des prix prohibitifs ( 3 à 6 dollars/ind.). Ce marché est du reste non négligeable puisque CULLEY (1978) l'estimait à 20 millions de grenouilles par an pour les USA. Par ailleurs, il existe aussi des élevages rustiques dans des parcs extérieurs, des étangs que l'on aménage par un entourage, une lutte antiprédateurs et avec un apport de nourriture aux têtards. Ce type de spéculation n'est réalisable qu'en climat tropical, avec de grosses espèces à croissance rapide, un coût de main-d'œuvre réduit au maximum et une rentabilité aléatoire (Brésil, Java,...). C'est pourquoi tous les animaux importés actuellement pour la consommation sont prélevés dans des populations sauvages (Indes, Indonésie....).

Les élevages présentent en éffet des difficultés à surmonter, comme la nourriture pour adultes qui doit être vivante (insectes, têtards...). Par contre. l'élevage intensif des têtards est possible, si une hygiène rigoureuse est respectée. Le taux de métamorphose (par rapport au nombre de têtards au départ) peut être élevé : 80 à $95 \%$ pour $R$. temporaria (quelques pour mille dans la nature) avec des densités de $5.000-6.000 / \mathrm{m}^{2}, 60$ à $70 \%$ pour $R$. esculenta avec une densité plus faible de $1.000 / \mathrm{m}^{2}$. L'élevage donne donc un taux de réussite incomparable par rapport à la nature, mais l'on ne connaît pas encore les taux de survie de ces grenouillettes replacées dans les conditions normales; ces résultats sont donc à accueillir avec prudence.

Pour le moment, aucune donnée précise de repeuplement n'est disponible dans la littérature. Des expériences en cours donnent quelques espoirs mais demandent du recul quant au maintien des populations implantées. A ce niveau, surtout en ce qui concerne les grenouilles vertes, il est impératif de prendre des précautions pour éviter toute déviation génétique. L'implantation doit non seulement concerner simultanément $R$. esculenta et $R$. lessonae mais aussi des animaux de même origine et les plus proches possible sur le plan géographique. Les contraintes réelles de telles opérations restent encore à définir.

En conclusion, une protection des zones à grenouilles contre les excès agricoles et urbains, des aménagements au niveau de la reproduction (frayères, passages sous les routes...) associés à une réglementation spécifique, claire, simple et respectée, sont actuellement les meilleurs moyens pour développer les populations existantes.

\section{REFERENCES BIBLIOGRAPHIQUES}

BEAUDOIN-BODIN J., MAILLARD Y., 1972. Batraciens et Reptiles de Brière. Penn ar bed, 8, 4, 407-414

BEEBEE T.J.C., 1973. Observations concerning the decline of the British Amphibia. Biol. Conserv., 5 , 20-24.

BEEBEE T.J.C., GRIFFIN J.R., 1977. Habitats of the British Amphibians. (1) Chalk Uplands. Biol. Conserv., 12, 279-293.

BEEBEE T.J.C., GRIFFIN J.R., 1977. A preliminary investigation into Natterjack toad (Bufo calamita) breeding site characteristics in Britain. J. Zool., Lond., 181, $341-350$.

BERGER L., 1964. Is Rana esculenta lessonae Camerano a distinct species ? Ann. Zool. 22, $245-261$.

BERGER L., 1966. Biometrical studies on the population of green frogs from the environs of Poznan. Ann. Zool., 23, 303-324. 
BERGER L., 1967. Embryonal and larval development of $F$, generation of green frogs from different combinations. Acta Zool. Crac., 12, 123-160

BERGER L., 1968. Morphology of the F, generation of various crosses within Rana esculenta complex. Acta Zool. Crac., 3, $301-324$

BERGER L., 1970. Some characteristics of the crosses within Rana esculenta complex in postlarval development. Ann. Zool., 27, 373-416

BERGER L., 1971 a Inheritance of sex and phenotype in $F_{1}$ and $F_{2}$ crosses within $R$. esculenta complex Genet. Pol., 12, 517.521

BERGER L., $1971 \mathrm{~b}$. Sex ratio in the $\mathrm{F}_{1}$ progeny within forms of Rana esculenta complex. Genet. Pol., 12, 87-101

BERGER L., 1971c. Viability, sex and morphology of $F_{2}$ generation within forms of Rana esculenta complex. Zool. Poloniae, 21, 345-391

BERGER L., 1973a. Systematics and hybridization in European green frogs of Rana esculenta complex. J. Herpetol. 7, 1 10.

BERGER L., 1979. Egg size as an index of phenotype in progeny of Rana esculenta females. Mitt. Zool. Mus. Berlin, 55, 187-202

BERGER L., 1983. Western Palearctic water frogs (Amphibia, Ranidae) : Systematics, genetics and population compositions. Experientia, 39, 127.130.

BERGER L., ROGUSKI H., 1978. Ploïdy of progeny from different egg size classes of Rana esculenta $L$. Folia Biol. (Krakow), 26, $231-248$.

BINKERT J., BORNER P. CHEN P.S., 1982. Rana esculenta complex: An experimental analysis of lethality and hybridogenesis. Experientia, 38, 1283-1292.

BLANKENHORN H.J., 1973. Zum Stand der Forschung über die Verbreitung der Grünfrösche im Kanton Zürich. Rev. Suisse Zool., 80, 655-662.

BLANKENHORN H.J., HEUSSER H., NOTTER P., 1973. Zum Verbreitung von R. lessonae im Kanton Zürich. Rev. Suisse Zool., 80, 662-666

BLANKENHORN H.J., HEUSSER H, VOGEL P., 1971. Drei Phänotypen von Grünfröschen aus dem Rana esculenta Komplex in der Schweiz. Rev. Suisse Zool., 78, 1243-1247.

COOKE A.S., 1970. The effect of pp'-DDT on tadpoles of the common frog (Ranatemporaria). Environ. Pollut., 1, 57-71.

COOKE A.S., 1973. Response of Rana temporaria tadpoles to chronic doses of pp'-DDT. Copeia, 4 , 647-652.

COOKE A.S., 1974. The effects of pp'-DDT on adult frogs (Rana temporaria). Brit. J. Herpetol., 5, 390-396.

COOKE A.S., 1977. Effects of field applications of the herbicides diquat and dichlobenil on Amphibians. Environ. Pollut., 12, 43-49

COOKE A.S., 1979. The influence of rearing density on the subsequent response to DDT dosing for tadpoles of the frog Rana temporaria. Bull. Environm. Contam. Toxicol., 21, 837-841

COOKE A.S., 1981. Tadpoles as indicators of harmful levels of pollution in the field. Environ. Pollut. Ser. A, 25, 123-133.

COOKE A.S., FERGUSON P.F., 1976. Changes in status of the frog (Rana temporaria) and the toad (Bufo bufo) on part of the East Anglian Fenland in Britain. Biol. Conserv.. 9, 191-198.

CULLEY D.D., HORSEMAN N.D., 1978. Current status of Amphibian culture with emphasis on nutrition, diseases and reproduction of the bullfrog, Rana catesbeiana in Proceed. 9th Annual Meet. World mariculture Soc.. Atlanta, J.W. AWAULT, Ed., 653-669

ENGELMAN"W.E., 1972. Disk-Elektrophorese der Serum proteine von Wasserfioschen. Ein Beitrag zum Diskussion über den Hybridcharakter von R. esculenta L. Acta Biol. Med. Germ., 29, $431-435$

ENGELMAN W.E., 1973. Zur Frage der verwandtschaftlichen Beziehungen europaischer Grünfrösche (Gattung Rana). Eine vergleichende elektrophoretische Untersuchung der Serumproteine. Zool. Jb. Syst., 100, 183-196

ENGELMAN W.E., 1974. Vergleichende Enzymuntersuchungen der Seren mitteleuropäischer Raniden (Amphibia, Anura). Experientia, 30, 870-872.

FLEURY J., L'HOSTIS D., 1977. Les marchés d'utilisation et de consommation des grenouilles en France (Rapt. INRA-Economie, Nantes), $24 \mathrm{p}$.

GIBBS E.L., NACE G.W., EMMONS M.B., 1971. The live frog is almost dead. Bio-Science, 21, 1027-1034 
GOSSEYE M. 1977. Etude de la dynamique d'une population de grenouilles Ranatemporaria en Haute Ardenne et des relations entre ces batraciens et l'environnement humain. Mémoire de Licence. Université de Louvain, $167 \mathrm{p}$.

GOSSEYE M., 1979. L'homme, prédateur des grenouilles dans le Luxembourg belge. Les Naturalistes belges, 60, 87-95

GRAF J.D.. MÜLLER W.P. 1979. Experimental gynogenesis provides evidence of hybridogenetic reproduction in the Rana esculenta complex. Experientia, 35, 1574-1576

GRAF J.D., KARCHF., MOREILLON M.C., 1977. Biochemical variation in the Rana esculenta complex A new hybrid form related to Rana perezi and Rana ridibunda. Experientia, 33, 1582-1584

GROSSENBACHER K., 1977. Die Amphibien des Kantons Bern. Mitt. Naturforsch. Ges. Bern. 34. $1-64$.

GÜNTHER R., 1970. Der Karyotyp von Rana ridibunda Pall. und das Vorkommen von Triploïdie bei Rana esculenta L. (Anura, Amphibia). Biol. Zbl., 89, 327-342.

GÜNTHER R., 1973. Uber die verwandtschaftlichen Beziehungen zwischen den europäischen Grünfröschen und den Bastardcharakter von Rana esculenta L. (Anura). Zool. Anz., 190, 250-285.

GÜNTHER R., 1975a. Untersuchungen der Meiose bei Männchen von Rana ridibunda Pall., Rana lessonae Cam. und der Bastardform, Rana esculenta L. (Anura). Biol. Zbl., 94, 277-294.

GÜNTHER R., 1975b. Zum natürlichen Vorkommen und zur Morphologie triploider Teichfrösche, Rana esculenta L. in der DDR (Anura, Ranidae). Mitt. Zool. Mus. Berlin, 51, 145-158

GÜNTHER R., 1979. General remarks on the evolutionary genetics of the European waterfrog complex. Mitt. Zool. Mus. Berlin, 55, 7-11.

GÜNTHER R., HÄHNEL S., 1976. Untersuchungen über den Genfluss zwischen Rana ridibunda und Rana lessonae sowie die Rekombinationsrate bei der Bastardform Rana esculenta (Anura. Ranidae). Zool. Anz., Jean, 197, 23-38.

GÜNTHER R., UZZELL T., BERGER L., 1979. Inheritance patterns in triploid Rana esculenta(Amphibia, Salientia). Mitt. Zool. Mus. Berlin, 55, 35-37

HALL R.J., KOLBE E., 1980. Bioconcentration of organophosphorus pesticides to hazardous levels by amphibians. J. Toxicol. and Environ. Health, 6, 853-860.

HEPPICH S., TUNNER H.G., GREILHUBER J., 1982. Premeïotic chromosome doubling after genome elimination during spermatogenesis of the species hybrid $R$. esculenta. Theor. Appl. Genet., 61, $101-104$.

HOTZ H., 1974. Ein Problem aus vielen Fragen - europaïsche Grünfrösche (Rana esculenta - Komplex) und ihre Verbreitung. Natur. Mus., 104, 262-272.

HOTZ H., UZZELL T., 1982. Biochemically detected sympatry of the water frog species : two different cases in the Adriatic Balkans (Amphibia, Ranidae). Proc. Acad. Nat. Sci. Philadelphia, 134, 50-79.

HOTZ H., UZZELL T., 1983. Interspecific hybrids of Rana ridibunda without germline exclusion of a parental genome. Experientia, 39, 538-540.

ILOSVAY G., 1977. Effect of urbanization on the herpetofauna of a settlement of the Tiscia (Szeged). Tiscia (Szeged), 12, 123-130.

KOREF-SANTIBANEF S., 1979. The karyotypes of Rana lessonae Cam. Rana ridibunda Pall. and the hybrid form Rana esculenta L. (Anura). Mitt. Zool. Mus. Berlin, 55, 115-124

LAUGHLIN R., LINDEN O., 1982. Sublethal responses of the tadpoles of the European frog Rana temporaria to two tributyltin compounds. Bull. Environm. Contam. Toxicol., 28, 494-499

LEUTSCHER A., 1975. An invader's decline and fall. The edible frog in England. Country life, 157. 455-456.

LYONS D.B., BUCKNER C.H., MCLEOD B.B., SUNDARAM K.M.S., 1976. The effects of fenitrothion, matacil and orthene on frog larvae. Chemical Control Research Institute, Ottawa, Ontario, 86 p.

MARCHAL-SEGAULT D., 1976. Toxicité de quelques insecticides pour des têtards de Bufo bufo (Amphibiens, Anoures). Bull. Ecol., 7. 411-416.

MARCHAL-SEGAULT D., RAMADE F., 1981. The effects of Lindane, an insecticide, on hatching and postembryonic development of Xenopus laevis (Daudin) Anuran Amphibian. Environm. Res., 24, 250-258.

MARION L.P., 1975. Contribution à l'étude écologique du lac de Grand-Lieu. Soc. Sci. Natur. de l'Ouest de la France. Museum d'histoire naturelle. Nantes, Suppl. hors série, $611 \mathrm{p}$

NEVEU A., 1980. L'élevage extensif des grenouilles. Perspectives et réalités in " La Pisciculture en étang ", R. BILLARD, ed., Public. INRA, Paris, 325-332 
OBERT H.J., 1977. Ökologische Unterschungen zum Rückgang von Froschlurchen in zwei Biotopen des Rein-Sieg-Gebiets zwischen 1971 und 1976. Salamandra, 13, 121-140.

OGIELSKA-NOWAK M., 1978. DNA content in erythrocyte nuclei of diploïd and triploïd green frog hybrids of Rana esculenta L. complex. Zool. Poloniae, 27, 109-115.

PAULOV S., 1977. Effect of the herbicide Gramoxone S (dichloride paraquat) on the development and body proteins of Amphibia (Rana temporaria L.) Biologia (Bratislava), 32, 127-131.

PAULOV S., 1981. Hazards of the fungicide Afugan (pyrasophos) for the development of Amphibia (Rana temporaria L.). Biologia (Bratislava), 36, 135-140.

REGNIER V., 1983. Etude écologique des populations de grenouilles du complexe Rana esculenta L. en pays de Vilaine (Bretagne). Thèse Doct. $3^{e}$ cycle, Univ. Rennes, $115 \mathrm{p}$.

SIMMS C., 1969. Indications of the decline of breeding amphibians at an isolated pond in marginal land, 1954-1967. Brit. J. Herpetol., 4, 93-96.

TERHIVUO J., 1981. Provisional atlas and population status of the Finnish amphibian and reptile species with reference to their ranges in northern Europe. Ann. Zool. Fennici, 18, 139-164.

TUNNER H.G., 1970. Das Serumeiweissbild einheimischen Wasserfrösche und der hybrid Charakter von Rana esculenta. Verh. Disch. Zool. Ges., 64, 352-358.

TUNNER H.G., 1972. Serologische und morphologische Untersuchungen zur Frage der Artabgrenzung bei Wasserfröschen aus der Umgebung von Mainz (Rhein-Main-Gebiet). Z. zool. Syst. Evolut.-forsch., 10,127-132.

TUNNER H.G., 1973a. Demonstration of the hybrid origin of the common green frog Rana esculenta L. Naturwiss, 10, $481-482$.

TUNNER H.G., 1973b. Das Albumin und andere Bluteiweisse bei Rana ridibunda Pallas, $R$. lessonae Camerano, $R$. esculenta Linné und deren Hybriden. Z. zool. Syst. Evolut. -forsch., 11, 219-233.

TUNNER H.G., 1974. Die klonale Struktur einer Wasserfrosch population. Z. zool. Syst. Evolut. -forsch., 12, 309-314.

TUNNER H.G., HEPPICH S., 1981. Premeiotic genome exclusion during oogenese in the common edible frog, Rana esculenta. Naturwiss, 68, 207-208.

TUNNER H.G., HEPPICH S., 1982. A genetic analysis of water frogs from Greece : evidence for the existence of a cryptic species. Z. zool. Syst. Evolut. -forsch., 20, 209-223.

TUNNER H.G., UZZELL T., 1974. Das Serumalbumin bei Rana ridibunda perezi(Salientia, Ranidae). Salamandra, 10, 137-139.

UZZELL T., 1973. Immunological distances between the serum albumins of Rana ridibunda and Rana lessonae. Proc. Acad. Nat. Sci. Philadelphia 130, 1-10.

UZZELL T., 1979. Immunological variation in Rana lessonae (Amphibia, Salientia). Mitt. Zool. Mus. Berlin, 55, 29-33.

UZZELL T., BERGER L., 1975. Electrophoretic phenotypes of Rana ridibunda, Rana lessonae and their hybridogenetic associate, Rana esculenta. Proc. Acad. Nat. Sci. Philadelphia, 127, 13-24.

UZZELL T., HOTZ H., 1979. Electrophoretic and morphological evidence for two forms of green frogs (Rana esculenta complex) in peninsular Italy (Amphibia, Salientia). Mitt. Zool. Mus. Berlin, 55, 13-27.

UZZELL T., BERGER L., GÜNTHER R., 1975. Diploïd and triploïd progeny from a diploïd female of Rana esculenta (Amphibia, Salientia). Proc. Acad. Nat. Sci. Philadelphia, 127, 81-91.

UZZELL T., GÜNTHER R., BERGER L., 1977. Rana ridibunda and Rana esculenta a leaky hybridogenetic system (Amphibia, Salientia). Proc. Acad. Nat. Sci. Philadelphia, 128, 147-171.

UZZELL T., HOTZ H., BERGER L., 1980. Genome exclusion in gametogenesis by an interspecific Rana hybrid : evidence from electrophoresis of individual oocytes. J. Exp. Zool., 214, 251-259.

VOGEL P., CHEN P.S., 1976. Genetic control of LDH isozymes in the Rana esculenta complex. Experientia, 32, 304-307.

WEIS J.S., 1975. The effect of DDT on tail regeneration in Rana pipiens and $R$. catesbeiana tadpoles. Copeia, 4, 765-767.

WIJNANDS, H.E.J., 1978. Plasma albumins and biometrical characteristics of different forms of Rana esculenta complex. Zool. Jb. Syst., 105, 337-346. 